\title{
Medical Image of the Week: Post-Anginal Sepsis Syndrome
}

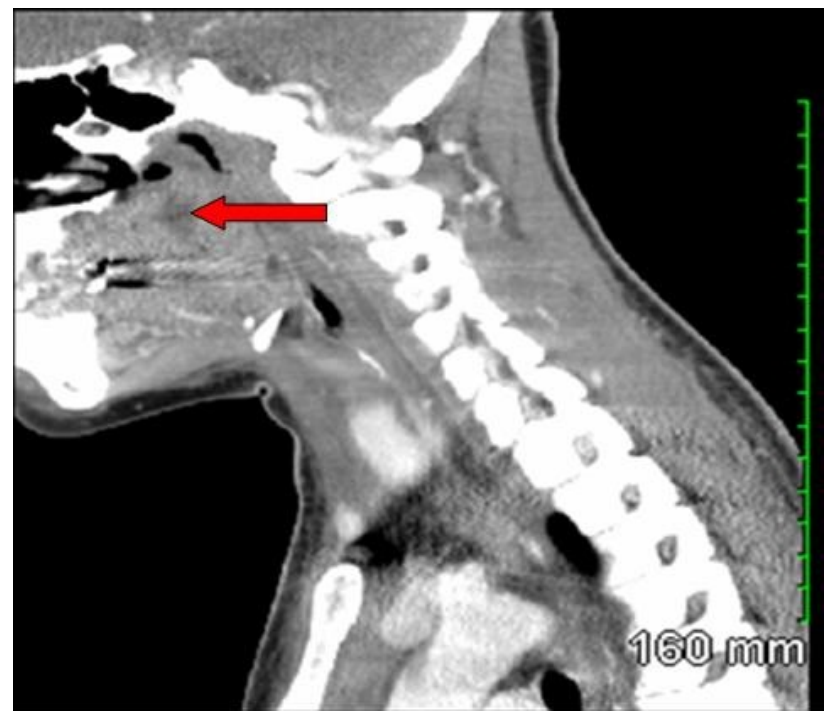

Figure 1. Computed tomography of soft tissue of neck showing enhancing fluid density (red arrow) within the left palatine tonsil compatible with peritonsillar abscess.

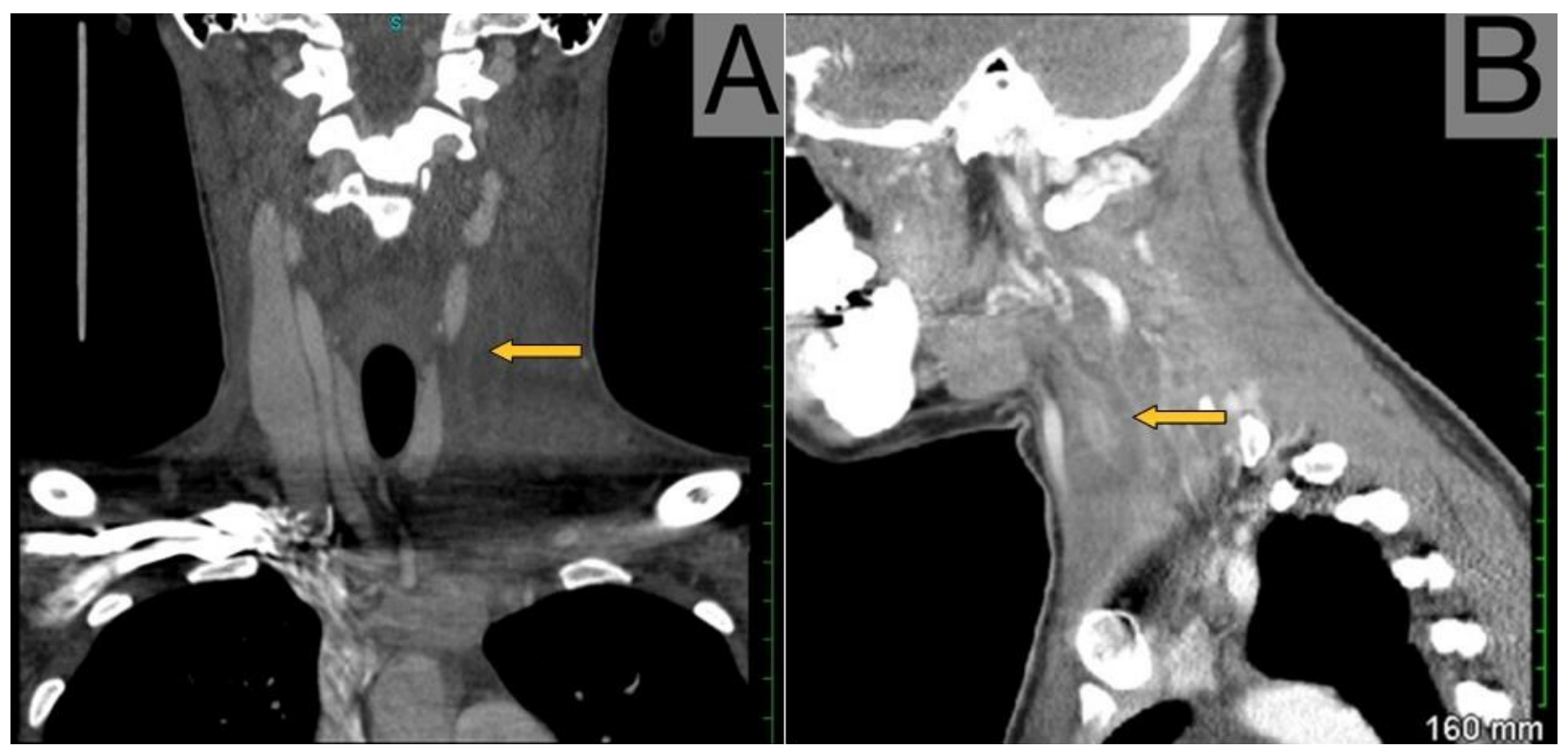

Figure 2. Anterior-posterior (panel A) and lateral (panel B) of the contrast-enhanced computed tomography of soft tissue of the neck showing filling defect throughout the entire left internal jugular vein from the skull base to its insertion at the left subclavian vein consistent with total occluding thrombus (yellow arrows).

A 22-year-old woman presented to our hospital with complaints of a persistent sore throat and intermittent low grade fever associated with chills for 10 days despite 5 days 
of antibiotics. During this time she had also developed progressive difficulty in swallowing due to associated pain that had progressed to limited mouth opening for past 2 days. Her vital signs were normal except for low grade fever. On limited oral cavity exam bilateral tonsils appeared enlarged and erythematous; tenderness was noted on palpation of left side of the antero-lateral neck with restriction of neck movements to the left. Basic labs revealed leukocytosis (WBC of $20.2 \mathrm{k} / \mathrm{mm}^{3}$ ) but was otherwise normal. Contrast-enhanced computed tomography of soft tissue of the neck was obtained which revealed bilaterally enlarged tonsils with small abscess within left palatine tonsil, filling defect throughout the entire left internal jugular vein from the skull base to its insertion at the left subclavian vein consistent with acute thrombosis (Figures 1 and 2). She improved considerably with intravenous antibiotics. Rapid strep test, blood and throat culture were negative. HIV, Epstein-Barr virus and cytomegalovirus antibodies were also negative. As she clinically improved we discharged her home with oral antibiotics and did not start her on anticoagulation.

Lemierre's syndrome is a septic thrombophlebitis of the internal jugular vein (IJV) commonly caused by anaerobic oro-pharyngeal flora usually by Fusobacteirum necrophorum although a wide range of bacteria may cause the syndrome $(1,2)$. Infection is usually followed by fulminant sepsis. The infection typically originates in the palatine tonsils or peritonsillar tissue which spreads into the lateral pharyngeal space causing septic thrombophlebitis of IJV which is usually followed by distal septic embolization, resulting in multi-organ involvement with lung being the most commonly affected. Diagnosis is usually established on the presence of thrombus in IJV and a positive blood culture, but cultures can be negative in about $12 \%$ of cases. Computed tomography of neck with contrast is the diagnostic modality of choice to demonstrate the thrombus. Prolonged course of Intravenous antibiotic (3-6 weeks) covering $F$. necrophorum and oral streptococci is the cornerstone of treatment. Currently there are no clear guidelines for the use of anticoagulation due to its rarity and lack of randomized controlled studies.

Chandramohan Meenakshisundaram MD, Nanditha Malakkla MD and Venu Ganipisetti, MD

Department of Internal Medicine, Presence Saint Francis Hospital

Evanston, IL

\section{References}

1. Srivali N, Ungprasert $P$, Kittanamongkolchai $W$, Ammannagari N. Lemierre's syndrome: An often missed life-threatening infection. Indian J Crit Care Med. 2014;18(3):170-2. [CrossRef] [PubMed]

2. Pinheiro PE, Miotto PD, Shigematsu NQ, Tamashiro E, Valera FC, Anselmo-Lima WT. Lemierre's syndrome: a pharyngotonsillitis complication. Braz J

Otorhinolaryngol. 2015;81(1):115-6. [CrossRef] [PubMed] 